\title{
Immunological Differences Between Right-Sided and Left- Sided Colorectal Cancers: A Comparison of Embryologic Midgut and Hindgut
}

\author{
Han Deok Kwak, Jae Kyun Ju \\ Department of Surgery, Chonnam National University Hospital, Gwangju, Korea
}

Purpose: There are known differences in embryology, clinical symptoms, incidences, molecular pathways involved, and oncologic outcomes of right-sided and left-sided colorectal cancers. However, immunologic study has only been characterized for healthy adults. The present study was designed to identify differences in immune cell populations in patients with right-sided and left-sided colorectal cancers.

Methods: A total of 35 patients who underwent colorectal resection for cancer between November 2016 and August 2017 at a tertiary teaching hospital were enrolled in this study. Patients were excluded if they had a disease affecting their immune system. Populations of immune cells, including mucosal-associated invariant T (MAIT), gamma delta T, invariant natural killer T, T, natural killer, and B cells, were measured in the peripheral blood and cancer tissues using flow cytometry, and then assessed based on the origin of the colorectal cancer.

Results: Fifteen had right-side and 20 had left-side colorectal cancer. There were no significant differences between the 2 cohorts for patient characteristics including pathologic stage. Peripheral blood from patients with right-side colon cancers contained fewer MAIT (0.87\% right-side vs. $1.74 \%$ left-side, $\mathrm{P}=0.028)$ and gamma delta $\mathrm{T}$ cells $(1.10 \%$ right-side vs. $3.05 \%$ left-side, $\mathrm{P}=0.002$ ). Although the group with right-side colorectal cancer had more MAIT cells in cancer tissues $(1.71 \%$ vs. $1.00 \%)$, this difference was not statistically significant.

Conclusion: There is a difference in population sizes of immune cells in blood between patients with right-sided and leftsided colon cancers. The immune cell composition was determined to be distinct based on embryologic origin.

Keywords: Colorectal cancer; Embryology; Sidedness; Immune cell

\section{INTRODUCTION}

During embryonic development, the colon originates from the midgut and hindgut, and becomes differentiated into the rightsided and left-sided colon based on the location. Tumors in the cecum, ascending colon, and proximal part of the transverse co-

Received: January 10, 2019 - Accepted: March 17, 2019

Correspondence to: Han Deok Kwak, M.D.

Department of Surgery, Chonnam National University Hospital,

42 Jebong-ro, Dong-gu, Gwangju 61469, Korea

Tel: +82-62-220-6456, Fax: +82-62-227-1635

E-mail: galaxy9135@naver.com

ORCID code: https://orcid.org/0000-0002-0716-6914

(C) 2019 The Korean Society of Coloproctology

This is an open-access article distributed under the terms of the Creative Commons Attribution NonCommercial License (http://creativecommons.org/licenses/by-nc/4.0) which permits unrestricted noncommercial use, distribution, and reproduction in any medium, provided the original work is properly cited. lon are defined as midgut tumors, while those in the distal transverse, descending, sigmoid colon, and rectum are considered hindgut tumors. Differences have been reported between rightsided and left-sided colon cancers for clinical symptoms [1], incidences [1], molecular pathways involved [2], and oncologic outcomes [1, 3-5], as well as embryologic origins. However, immunological differences in the right-sided and left-sided colons have only been reported in healthy adults [6]. Studies have shown the short- and long-term outcomes of colorectal cancers depend on the location of the cancer and, therefore, it is argued the treatment plans for these cancers should be distinct. Meanwhile, cells involved in mucosal immunity have been recently discovered [7]. Therefore, the present study aimed to identify immunological differences between patients with right-sided and left-sided colon cancers. 


\section{METHODS}

The patients enrolled in this study were aged at least 18 years and diagnosed with colorectal cancer. Patients with diseases that could directly affect or that require treatments that affect immune cell populations, such as unregulated diabetes, tuberculosis, autoimmune diseases, and treatments involving immunosuppressants, were excluded. The embryologic boundary of sidedness has not been clearly defined. Therefore, patients with transverse colon, mid- and lower rectal cancers were also excluded. A total of 35 patients were included in this study and were enrolled between November 2016 and August 2017.

Patients' clinical data, including age, sex, body mass index, American Society of Anesthesiologists physical status classification, previous medical history, surgical history, operation duration, and volume of estimated blood loss were analyzed and compared between cohorts. Pathologic outcomes assessed were tumor size and stage, including lymph node status. Postoperative outcomes, including days to bowel movement and diet resumption, length of hospital stay, and postoperative complications, were also compared between the groups.

The immune cell populations were measured based on the presence of cell surface antigens using a cell analyzer and the Kaluza program by separating peripheral blood mononuclear cells (PBMCs) from peripheral blood and cancer tissue samples, staining for 20 minutes with various monoclonal antibodies, and then analyzing by flow cytometry. The immune cells in the colonic mucosa included mucosal-associated invariant T (MAIT), gamma delta $(\gamma \delta) \mathrm{T}$, invariant natural killer T (iNKT), T, natural killer (NK), and B cells. Immune cells are known to be difficult to store because their phenotype may change when exposed to changes in temperature. Therefore, peripheral blood collected just before surgery was immediately transferred aseptically to the laboratory for analysis.

PBMCs were isolated by density-gradient centrifugation with Ficoll-Paque Plus solution (Amersham Bioscience, Uppsala, Sweden) using peripheral venous blood samples collected in heparincontaining tubes. Total lymphocyte numbers were measured by Coulter LH750 automatic hematology analyzer (Beckman Coulter, Miami, FL, USA). MAIT cells were phenotypically identified by flow cytometry using CD3+TCR $\gamma \delta-\mathrm{V} a 7.2+\mathrm{CD} 161^{\text {high }}$. The absolute number of MAIT cells was obtained by multiplying the total lymphocyte number per microliter and the percentage of the $\mathrm{CD} 3+\gamma \delta$-T cells in MAIT cell. PBMCs were stained with PE-conjugated anti-CD3, FITC-conjugated anti-TCR $\gamma \delta$, APC-conjugated anti-TCR Va7.2, and PE-Cy5-conjugated anti-CD161 to sort the MAIT cells. Then, CD3+TCR $\gamma \delta-V \alpha 7.2+C D 161^{\text {high }}$ MAIT cells were obtained with FACS Aria I sorter (BD Biosciences, Mountain View, CA, USA). The MAIT cells were separated over $98 \%$ purity with this process. The cancer tissues were taken from the central lesion such as fungating or ulcero-fungating area with a size of $1 \mathrm{~cm} \times 1 \mathrm{~cm}$ without including the deep area for ver- ification of stage T. Single-cell suspension was prepared on $40-\mu \mathrm{m}$ cell strainers within 30 minutes after resection from patients for flow cytometry. The specimen was fixed with neutral buffered formalin, then was embedded in paraffin for immunofluorescence staining. Immune cells were analyzed and compared in the peripheral venous blood, however, cancer tissues were only analyzed those being showed difference in blood samples.

All patients were fully informed and consent was obtained prior to enrollment in this study. This study was approved by the Institutional Review Board (CNUH-2017-066) at Chonnam National University Hospital.

\section{Statistical analysis}

Differences between groups were assessed using Student t-test or the Mann-Whitney U-test for continuous data and the chi-square or Fisher exact test for categorical data. Statistical analyses were performed using IBM SPSS Statistics ver. 20.0 (IBM Co., Armonk, NY, USA). A P-value $\leq 0.05$ was considered statistically significant.

\section{RESULTS}

\section{Patient characteristics and perioperative results}

Of the 35 patients enrolled in this study, 15 (42.9\%) had rightand 20 had left-sided colon cancer. The following parameters were analyzed, but did not differ significantly between the groups: mean age, sex, body mass index, American Society of Anesthesiologists physical status classification, past medical history, previous abdominal operative history, and preoperative carcinoembryonic antigen level (Table 1). Surgical factors, such as operative methods and average operating time, did not differ between the 2 groups. The average length of hospital stay (overall average, 8.4 days; 9 days right-side vs. 8.2 days left-side, $\mathrm{P}=0.771$ ), complications during hospitalization, and pathologic stage $(\mathrm{P}=0.379)$ of the patients were also not statistically different (Table 2).

\section{Mucosal immune cell populations}

Peripheral blood from patients with right-side colon cancers contained fewer MAIT (0.87\% right-side vs. $1.74 \%$ left-side, $\mathrm{P}=$ $0.028)$ and $\gamma \delta$ T cells (1.10\% right-side vs. 3.05\% left-side, $\mathrm{P}=$ 0.002 ). No significant differences were observed in the other immunologic cell types assessed: iNKT, T, NK, and B cells. While the patients with right-side cancer had more MAIT cells in cancer tissues $(1.71 \%$ right-side vs. $1.00 \%$ left-side, $\mathrm{P}=0.256)$, this difference was not statistically significant (Table 3 ).

\section{DISCUSSION}

In the present study, it was found mucosal-associated immune cells, such as MAIT and $\gamma \delta$ T cells, had differences in distribution based on whether patients had right- or left-side tumors. A 2015 review [2] reported they had differences such as frequency, histol- 
Table 1. Patients' characteristics

\begin{tabular}{|c|c|c|c|}
\hline Characteristic & Right & Left & P-value \\
\hline Age (yr) & $66.73 \pm 2.27$ & $64.43 \pm 2.05$ & 0.493 \\
\hline Sex & & & 0.828 \\
\hline Male & $9(60)$ & $19(63.3)$ & \\
\hline Female & $6(40)$ & $11(36.7)$ & \\
\hline Body mass index (kg/m²) & $22.55 \pm 0.95$ & $23.31 \pm 0.59$ & 0.483 \\
\hline ASA PS classification & & & 0.659 \\
\hline I & $3(20)$ & $5(16.5)$ & \\
\hline$\|$ & $11(73.3)$ & $20(67)$ & \\
\hline III & $1(6.7)$ & $5(16.5)$ & \\
\hline Medical history & & & 0.783 \\
\hline \multicolumn{4}{|l|}{ Yes } \\
\hline Endocrine disease (DM) & $3(20)$ & $5(16.7)$ & \\
\hline Cardiac disease (HTN) & $8(53)$ & $14(46.7)$ & \\
\hline Pulmonary disease & $1(6.7)$ & $16(53.3)$ & \\
\hline No & $3(20)$ & $5(16.7)$ & \\
\hline Previous abdominal operative history & $6(40)$ & $15(50)$ & 0.526 \\
\hline \multicolumn{4}{|l|}{ Substance use } \\
\hline Smoking & 2 (18.2) & $5(23.8)$ & 0.715 \\
\hline Alcohol & $3(27.3)$ & $6(28.6)$ & 0.938 \\
\hline Preop CEA (ng/mL) & $13.7 \pm 5.9$ & $9.48 \pm 3.6$ & 0.515 \\
\hline
\end{tabular}

Values are presented as mean \pm standard deviation or number (\%).

AV, anal verge; ASA PS, American Society of Anesthesiologists physical status; DM, diabetes mellitus; HTN, hypertension; Preop CEA, preoperative carcinoembryonic antigen.

ogy, oncology, and immunology depending on the location of the cancer. However, immune factors have only been compared in the right-sided and left-sided colon in healthy adults [6], not in patients with colon cancer. Therefore, to the best of our knowledge, this is the first report to assess distribution differences in immune cells in patients based on the side of colon cancer location. Studies of sidedness have only identified differences in outcomes and reactions to chemotherapy. In addition, these findings are controversial because bias may have been introduced through range of resection. The present study may reveal fundamental differences between right-sided and left-sided colorectal cancers because it was carried out preoperatively or before administration of chemotherapy. It is expected this study will serve as the basis for further studies into the antitumor effect of immune cells and ongoing research on functional differences. In addition, future work may include identifying biologic markers for disease prediction, evaluating response to adjuvant treatments, and determining prognosis of patients with colorectal cancer.

The outcomes on sidedness of colorectal cancer are still debatable. The American College of Surgeons National Surgical Quality Improvement Program database showed no differences in
Table 2. Operative and postoperative variables

\begin{tabular}{|c|c|c|c|}
\hline & Right & Left & P-value \\
\hline \multicolumn{4}{|l|}{ Diagnosis } \\
\hline Ascending colon & $13(86.7)$ & - & \\
\hline Hepatic flexure colon & $1(6.7)$ & - & \\
\hline Proximal transverse colon & $1(6.7)$ & - & \\
\hline Splenic flexure colon & - & $1(3.3)$ & \\
\hline Descending colon & - & $1(3.3)$ & \\
\hline Sigmoid colon & - & $20(66.7)$ & \\
\hline Rectosigmoid junction & - & $3(10)$ & \\
\hline Upper rectum & - & $5(16.7)$ & \\
\hline Operative methods & & & 0.053 \\
\hline Open & $12(80)$ & $15(50)$ & \\
\hline Laparoscopy & $3(20)$ & $15(50)$ & \\
\hline Operating time (min) & $104.4 \pm 13.7$ & $125.7 \pm 8.0$ & 0.161 \\
\hline Staging & & & 0.379 \\
\hline 0 & $3(20)$ & $1(3.3)$ & \\
\hline 1 & $4(26.7)$ & $6(20)$ & \\
\hline$\|$ & $4(26.7)$ & $10(33.3)$ & \\
\hline III & $3(20)$ & $10(33.3)$ & \\
\hline IV & $1(6.7)$ & $3(10)$ & \\
\hline Discharge (day) & $9.0 \pm 2.1$ & $8.2 \pm 1.6$ & 0.771 \\
\hline \multicolumn{4}{|l|}{ Postop complications } \\
\hline Postoperative ileus & $3(20)$ & $6(20)$ & 1 \\
\hline Urinary incontinence & $3(20)$ & $4(13.3)$ & 0.561 \\
\hline Surgical site infection & $2(13.3)$ & $3(10)$ & 0.737 \\
\hline
\end{tabular}

Values are presented as number (\%) or mean \pm standard deviation.

Table 3. Immunologic cell analysis

\begin{tabular}{lrrc}
\hline Variable & \multicolumn{1}{c}{ Right } & Left & P-value \\
\hline MAIT cell, blood (\%) & $0.87 \pm 0.18$ & $1.74 \pm 0.34$ & 0.028 \\
$\gamma \delta$ T cell, blood (\%) & $1.10 \pm 0.24$ & $3.05 \pm 0.52$ & 0.002 \\
iNKT cell, blood (\%) & $0.15 \pm 0.29$ & $0.16 \pm 0.20$ & 0.915 \\
T cell, blood (\%) & $59.01 \pm 4.98$ & $56.78 \pm 2.94$ & 0.683 \\
NK cell, blood (\%) & $27.95 \pm 5.76$ & $33.90 \pm 3.33$ & 0.346 \\
B cell, blood (\%) & $11.08 \pm 2.32$ & $8.25 \pm 1.92$ & 0.370 \\
MAIT cell, tissue (\%) & $1.71 \pm 0.56$ & $1.00 \pm 0.11$ & 0.256
\end{tabular}

Values are presented as mean \pm standard deviation.

MAIT, mucosal-associated invariant $\mathrm{T} ; \gamma \delta \mathrm{T}$, gamma delta T; iNKT, invariant natural killer; NK, natural killer.

comparison of short-term outcomes between the groups only with the exception of the incidence of superficial surgical site infection, which was more prevalent in left-side colon cancer [8]. In 2011, the Surveillance, Epidemiology, and End Results Program 
database of 53,801 patients found patients with left-side colorectal cancer had high mortality when cancer was stage II compared to stage III for patients' right-side colon cancer. However, there was no difference in the 5-year survival rate between these cohorts [9]. In 2014, a Japanese study with retrospective assessment of 820 patients for 17 years observed patients with right-side colon cancer had a better 5 -year disease-free survival $(\mathrm{P}=0.034)$ for stage I [5]. However, many studies have reported higher stages and poorer survival rates for right-side colon cancers. A study of 3,552 patients identified a difference in cancer incidence for a specific sex and age and found right-side colon cancer was more likely to be at a higher stage $(\mathrm{P}<0.001)$ than left-side colon cancer. In this study, the authors concluded the flat tumor type associated with right-side colon cancer and polypoid tumor type associated with left-side colon cancer may be related to different mechanisms of tumor formation [1]. A Surveillance, Epidemiology, and End Results Program database-based study published in 2008 on 77,978 patients also found poorer prognoses for patients with right-side colon cancers than left-side cancers $(\mathrm{P}<0.001)$ and hypothesized biological and environmental factors caused this difference [3]. A 2010 German study on 17,641 patients reported right-side colon cancers were more likely to be poorly differentiated or locally advanced and were associated with a lower survival rate $(\mathrm{P}<0.01)$. It was also found that left-side colon cancers had more frequent metastasis to the lungs and liver, while right-side colon cancers more metastasized to the abdominal cavity [4]. Based on a population-based database released in Australia in 2015, the overall survival rate is lower in patients with right-side colon cancer than left-side $(\mathrm{P}<0.001)[10]$.

Studies on the roles of immune cells in colon cancer are frequently being published. According to a study published in 2012 using the mouse colitis-associated colon cancer model, iNKT cells inhibit tumor progression [11]. A study aiming to decrease colorectal metastasis in mice found more tumor-infiltration of activated CD8+ T cells and fewer regulatory T cells in the spleen when interleukin (IL)-17 and oxaliplatin were injected simultaneously [12]. The authors concluded IL-7 changed cell sensitivity to oxaliplatin, but the results suggest it prevented tumor cell growth rather than being directly cytotoxic. Since MAIT cells were first described in 2003 [7], several studies have reported on their functions. MAIT cells are $\alpha \beta \mathrm{T}$ cells, are relatively abundant in humans, frequent expressers of TCR Va7.2-Ja33 and CD161, and make up approximately $10 \%$ of peripheral blood T cells, $10 \%$ of lamina propria lymphocytes, and $50 \%$ of hepatic $\mathrm{T}$ cells [13]. After activation by antigen, MAIT cells secrete T-helper $1 / 17$ cytokines, such as interferon-gamma (IFN- $\gamma$ ) and IL-17, in large quantities in a manner similar to an innate immune response or secrete granzyme B and/or perforin to remove pathogens $[14,15]$. The role of MAIT cells in colon cancers has not yet been characterized, but it is known IL-17 drives the inflammation that facilitates tumor progression and IFN- $\gamma$ is essential for antitumor immunity; tumor-associated MAIT cells cause antitumor immune responses through cytokine secretion. In this study, MAIT cells were found to be important in controlling host responses to infections, autoimmune diseases, and cancers [16]. In an in vitro study, MAIT cells were found to be present in colon cancer tissues, but had reduced production of IFN- $\gamma$ [17]. Another study reported a decrease in circulating memory CD8+ MAIT cells in advanced colon cancers and an increase in tumor-infiltrating MAIT cells. In particular, tumor-infiltrating MAIT cells were positively correlated with serum carcinoembryonic antigen levels $(\mathrm{R}=0.5971)$ and negatively correlated with the size of the circulating MAIT cell population $(\mathrm{R}=-0.5559)$ [18]. A follow-up study also evaluated the distribution of circulating and tumor-infiltrating MAIT cells in colon cancers and found activated circulating MAIT cells were involved in immune surveillance and acted by activating IL17 and reducing IFN- $\gamma$ production [19].

The present study has several limitations. Previously published related studies, including by the same authors, found the distribution of the circulating MAIT cells in the peripheral blood generally had an inverse correlation with the size of the MAIT cell tissue population [19]. It is possible the number of patients analyzed was not sufficient to reveal a statistically significant difference in tissue MAIT cells. It is also necessary to analyze more patients to assess differences in the distribution of immunologic cells for each stage of colon cancer. Second, the borders based on the origin of the colon are not clearly defined. The boundary between the midgut and hindgut is theoretically the distal one-third of transverse colon, but this is not fully accepted. Therefore, the authors excluded ambiguous boundaries, including the transverse colon, below the mid-rectum, and between the hindgut and cloaca, from this study.

In conclusion, the proportion of specific immune cells present in patients with colorectal cancers with distinct embryologic origins was confirmed to be different.

\section{CONFLICT OF INTEREST}

No potential conflict of interest relevant to this article was reported.

\section{ACKNOWLEDGMENTS}

This study was supported by a grant (CRI 17002-1) from the Chonnam National University Hospital Biomedical Research Institute.

\section{REFERENCES}

1. Nawa T, Kato J, Kawamoto H, Okada H, Yamamoto H, Kohno H, et al. Differences between right-sided and left-sided colon cancer in patient characteristics, cancer morphology and histology. J Gastroenterol Hepatol 2008;23:418-23.

2. Lee GH, Malietzis G, Askari A, Bernardo D, Al-Hassi HO, Clark 
SK. Is right-sided colon cancer different to left-sided colorectal cancer? - a systematic review. Eur J Surg Oncol 2015;41:300-8.

3. Meguid RA, Slidell MB, Wolfgang CL, Chang DC, Ahuja N. Is there a difference in survival between right- versus left-sided colon cancers? Ann Surg Oncol 2008;15:2388-94.

4. Benedix F, Kube R, Meyer F, Schmidt U, Gastinger I, Lippert H, et al. Comparison of 17,641 patients with right-sided and left-sided colon cancer: differences in epidemiology, perioperative course, histology, and survival. Dis Colon Rectum 2010;53:57-64.

5. Moritani K, Hasegawa H, Okabayashi K, Ishii Y, Endo T, Kitagawa Y. Difference in the recurrence rate between right-sided and left-sided colon cancer: a 17-year experience at a single institution. Surg Today 2014;44:1685-91.

6. Kirby JA, Bone M, Robertson H, Hudson M, Jones DE. The number of intraepithelial $\mathrm{T}$ cells decreases from ascending colon to rectum. J Clin Pathol 2003;56:158.

7. Treiner E, Duban L, Bahram S, Radosavljevic M, Wanner V, Tilloy F, et al. Selection of evolutionarily conserved mucosal-associated invariant T cells by MR1. Nature 2003;422:164-9.

8. Kwaan MR, Al-Refaie WB, Parsons HM, Chow CJ, Rothenberger DA, Habermann EB. Are right-sided colectomy outcomes different from left-sided colectomy outcomes?: study of patients with colon cancer in the ACS NSQIP database. JAMA Surg 2013;148: 504-10.

9. Weiss JM, Pfau PR, O'Connor ES, King J, LoConte N, Kennedy G, et al. Mortality by stage for right- versus left-sided colon cancer: analysis of surveillance, epidemiology, and end results--Medicare data. J Clin Oncol 2011;29:4401-9.

10. Price TJ, Beeke C, Ullah S, Padbury R, Maddern G, Roder D, et al. Does the primary site of colorectal cancer impact outcomes for patients with metastatic disease? Cancer 2015;121:830-5.

11. Yoshioka K, Ueno Y, Tanaka S, Nagai K, Onitake T, Hanaoka R, et al. Role of natural killer T cells in the mouse colitis-associated colon cancer model. Scand J Immunol 2012;75:16-26.

12. Gou HF, Huang J, Shi HS, Chen XC, Wang YS. Chemo-immunotherapy with oxaliplatin and interleukin-7 inhibits colon cancer metastasis in mice. PLoS One 2014;9:e85789.

13. Treiner E, Lantz O. CD1d- and MR1-restricted invariant T cells: of mice and men. Curr Opin Immunol 2006;18:519-26.

14. Le Bourhis L, Guerri L, Dusseaux M, Martin E, Soudais C, Lantz O. Mucosal-associated invariant T cells: unconventional development and function. Trends Immunol 2011;32:212-8.

15. Ussher JE, Klenerman P, Willberg CB. Mucosal-associated invariant T-cells: new players in anti-bacterial immunity. Front Immunol 2014;5:450.

16. Park YW, Kee SJ. Mucosal-associated invariant T cells: a new player in innate immunity. J Rheum Dis 2015;22:337-45.

17. Sundström P, Ahlmanner F, Akéus P, Sundquist M, Alsén S, Yrlid $\mathrm{U}$, et al. Human mucosa-associated invariant $\mathrm{T}$ cells accumulate in colon adenocarcinomas but produce reduced amounts of IFN- $\gamma$. J Immunol 2015;195:3472-81.

18. Ling L, Lin Y, Zheng W, Hong S, Tang X, Zhao P, et al. Circulating and tumor-infiltrating mucosal associated invariant T (MAIT) cells in colorectal cancer patients. Sci Rep 2016;6:20358.

19. Won EJ, Ju JK, Cho YN, Jin HM, Park KJ, Kim TJ, et al. Clinical relevance of circulating mucosal-associated invariant $\mathrm{T}$ cell levels and their anti-cancer activity in patients with mucosal-associated cancer. Oncotarget 2016;7:76274-90. 